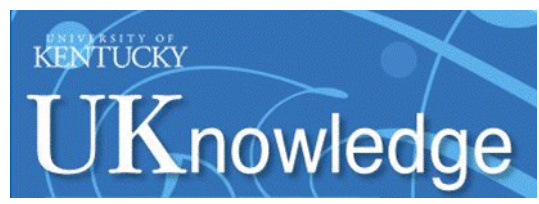

University of Kentucky

UKnowledge

4-1-2016

\title{
Spatio-Temporal Linear Stability Analysis of Stratified Planar Wakes: Velocity and Density Asymmetry Effects
}

\author{
Benjamin Emerson \\ Georgia Institute of Technology \\ Swapnil Jagtap \\ Georgia Institute of Technology \\ J. Mathew Quinlan \\ Georgia Institute of Technology \\ Michael W. Renfro \\ University of Kentucky, michael.renfro@uky.edu \\ Baki M. Cetegen \\ University of Connecticut - Storrs
}

See next page for additional authors

Follow this and additional works at: https://uknowledge.uky.edu/me_facpub

Part of the Fluid Dynamics Commons, and the Mechanical Engineering Commons

Right click to open a feedback form in a new tab to let us know how this document benefits you.

\section{Repository Citation}

Emerson, Benjamin; Jagtap, Swapnil; Quinlan, J. Mathew; Renfro, Michael W.; Cetegen, Baki M.; and Lieuwen, Tim, "Spatio-Temporal Linear Stability Analysis of Stratified Planar Wakes: Velocity and Density Asymmetry Effects" (2016). Mechanical Engineering Faculty Publications. 29.

https://uknowledge.uky.edu/me_facpub/29

This Article is brought to you for free and open access by the Mechanical Engineering at UKnowledge. It has been accepted for inclusion in Mechanical Engineering Faculty Publications by an authorized administrator of UKnowledge. For more information, please contact UKnowledge@lsv.uky.edu. 
Spatio-Temporal Linear Stability Analysis of Stratified Planar Wakes: Velocity and Density Asymmetry Effects

Digital Object Identifier (DOI)

https://doi.org/10.1063/1.4943238

Notes/Citation Information

Published in Physics of Fluids, v. 28, issue 4, 045101, p. 1-15.

(C) 2016 AIP Publishing LLC.

This article may be downloaded for personal use only. Any other use requires prior permission of the author and AIP Publishing.

The following article appeared in Physics of Fluids 28, 045101 (2016) and may be found at https://doi.org/10.1063/1.4943238

\section{Authors}

Benjamin Emerson, Swapnil Jagtap, J. Mathew Quinlan, Michael W. Renfro, Baki M. Cetegen, and Tim Lieuwen 


\section{Spatio-temporal linear stability analysis of stratified planar wakes: Velocity and density asymmetry effects}

Benjamin Emerson, Swapnil Jagtap, J. Mathew Quinlan, Michael W. Renfro, Baki M. Cetegen, and Tim Lieuwen

Citation: Physics of Fluids 28, 045101 (2016);

View online: https://doi.org/10.1063/1.4943238

View Table of Contents: http://aip.scitation.org/toc/phf/28/4

Published by the American Institute of Physics

\section{Articles you may be interested in}

Turbulence generation through intense kinetic energy sources

Physics of Fluids 28, 065106 (2016); 10.1063/1.4954052

Streak instability and generation of hairpin-vortices by a slotted jet in channel crossflow:

Experiments and linear stability analysis

Physics of Fluids 28, 014103 (2016); 10.1063/1.4939717

Scalar dissipation rate statistics in turbulent swirling jets

Physics of Fluids 28, 075104 (2016); 10.1063/1.4954657

Critical inclination for absolute/convective instability transition in inverted falling films

Physics of Fluids 28, 044107 (2016); 10.1063/1.4946827

Resonances in the forced turbulent wake past a 3D blunt body

Physics of Fluids 28, 065104 (2016); 10.1063/1.4953176

Round impinging jets with relatively large stand-off distance

Physics of Fluids 28, 075107 (2016); 10.1063/1.4955167

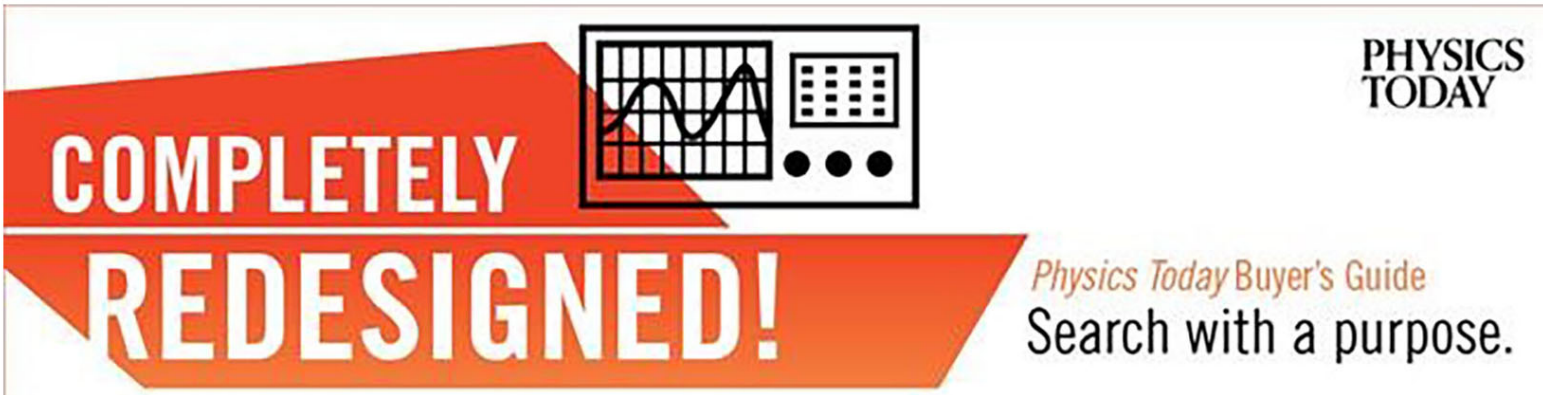




\title{
Spatio-temporal linear stability analysis of stratified planar wakes: Velocity and density asymmetry effects
}

\author{
Benjamin Emerson, ${ }^{1, a), b)}$ Swapnil Jagtap, ${ }^{1, b)} \mathrm{J}$. Mathew Quinlan, ${ }^{1}$ \\ Michael W. Renfro, ${ }^{2, c)}$ Baki M. Cetegen, ${ }^{3}$ and Tim Lieuwen ${ }^{1}$ \\ ${ }^{1}$ Department of Aerospace Engineering, Georgia Institute of Technology, Atlanta, \\ Georgia 30332, USA \\ ${ }^{2}$ Department of Mechanical Engineering, University of Kentucky, Lexington, \\ Kentucky 40506, USA \\ ${ }^{3}$ Department of Mechanical Engineering, University of Connecticut, Storrs, \\ Connecticut 06269, USA
}

(Received 21 August 2015; accepted 19 February 2016; published online 1 April 2016)

\begin{abstract}
This paper explores the hydrodynamic stability of bluff body wakes with non-uniform mean density, asymmetric mean density, and velocity profiles. This work is motivated by experiments [S. Tuttle et al., "Lean blow off behavior of asymmetrically-fueled bluff body-stabilized flames," Combust. Flame 160, 1677 (2013)], which investigated reacting wakes with equivalence ratio stratification and, hence, asymmetry in the base flow density profiles. They showed that highly stratified cases exhibited strong, narrowband oscillations, suggestive of global hydrodynamic instability. In this paper, we present a local hydrodynamic stability analysis for non-uniform density wakes that includes base flow asymmetry. The results show that increasing the degree of base density asymmetry generally has a destabilizing effect and that increasing base velocity asymmetry tends to be stabilizing. Furthermore, we show that increasing base density asymmetry slightly decreases the absolute frequency and that increasing the base velocity asymmetry slightly increases the absolute frequency. In addition, we show that increasing the degree of base density asymmetry distorts the most absolutely unstable hydrodynamic mode from its nominally sinuous structure. This distorted mode exhibits higher amplitude pressure and velocity oscillations near the interface with the smaller density jump than near the one with the bigger density jump. This would then be anticipated to lead to strongly non-symmetric amplitudes of flame flapping, with much stronger flame flapping on the side with lower density ratio. These predictions are shown to be consistent with experimental data. These comparisons support the analytical predictions that increased base density asymmetry are destabilizing and that hydrodynamic velocity fluctuation amplitudes should be greatest at the flame with the lowest density jump. (C) 2016 AIP Publishing LLC. [http://dx.doi.org/10.1063/1.4943238]
\end{abstract}

\section{BACKGROUND}

This paper describes an analysis of the hydrodynamic stability of reacting bluff body wakes. The bluff body wake is a common mode of flame stabilization in a variety of practical combustion devices. ${ }^{1}$ The unsteady flow fields of reacting bluff body wakes are often dominated by large scale coherent structures, embedded upon a background of acoustic waves and broadband fine scale turbulence. These large scale structures play important roles in such processes as combustion instabilities, ${ }^{2-6}$

\footnotetext{
a) Author to whom correspondence should be addressed. Electronic mail: bemerson@gatech.edu

b) B. Emerson and S. Jagtap contributed equally to this work.

c) This research was performed while M. Renfro was at the Department of Mechanical Engineering, University of Connecticut, Storrs, Connecticut 06269, USA.
} 
mixing and entrainment, flashback, and blow off, ${ }^{1}$ and they arise because of underlying hydrodynamic instabilities of the flow field. ${ }^{7}$

We quickly review key dynamical features of non-reacting wake flows. A pair of separating shear layers appears immediately downstream of the bluff body in high Reynolds number, planar bluff body flows. ${ }^{8}$ These shear layers are unstable due to the Kelvin-Helmholtz mechanism, ${ }^{9}$ and they roll up into spatially concentrated locations of high vorticity and grow in thickness. ${ }^{10,11}$ In uniform density flows, the growing shear layers interact strongly, resulting in an unstable, sinuous roll up of the bluff body wake into large-scale vortical structures. ${ }^{12}$ This instability manifests itself as the Von Karman vortex street and has a characteristic frequency ${ }^{9}$ of

$$
f_{b v k}=S t \frac{U_{u}}{D}
$$

where $U_{u}$ is the lip velocity at the bluff body, $D$ is the cross-stream diameter of the bluff body, and $S t$ is the Strouhal number. For circular cylinders, $S t$ is independent of Reynolds number $(S t=0.21)$ in the turbulent shear layer, laminar boundary layer regime, i.e., at Reynolds numbers in the range of $\sim 1000<\operatorname{Re}<\sim 200000 .{ }^{13}$ Bluff body shape also influences the Strouhal number for the Von Karman vortex street. ${ }^{14}$ In particular, $S t$ is lower for "bluffer" bodies, i.e., those with higher drag and wider wakes. ${ }^{15,16}$

Experimental studies of reacting bluff body wakes have demonstrated that combustion often tends to suppress the sinuous wake instability. ${ }^{17-19}$ For example, Erickson et al. ${ }^{19}$ performed a systematic study that illustrated the influence of the flame density ratio upon the large scale flow dynamics. Their results, reproduced in Figure 1, show that a large sinuous flow feature becomes prominent as the density ratio across the flame is reduced below a value of approximately 2 . Similarly, Emerson et al. ${ }^{20}$ experimentally explored the role of the flame density ratio, $\rho_{u} / \rho_{b}$, where $\rho_{u}$ is the reactant (un-burned) density and $\rho_{b}$ is the product (burned) density. They observed a gradual wake structure transition in a density ratio range of roughly $2-3$. This observation is quite significant as it demonstrates that the dominant fluid mechanics in a burner with non-preheated reactants, which has a "high" density ratio, can be very different from those of a facility with highly preheated reactants, such as many industrial combustion applications. For example, stoichiometric methane air flames with reactant temperatures of $300 \mathrm{~K}$ and $1000 \mathrm{~K}$ have density ratios of 7.5 and 2.6, respectively.

These fundamentally different wake dynamics observed at high and low flame density ratios are the manifestations of different instabilities. The low density ratio scenario (see Figure 1(a)) is a globally unstable flow, while the high density ratio scenario (see Figure 1(b)) is a convectively unstable flow. Convectively unstable flows have positive spatial growth rates for one or more disturbance wavelengths with nonzero group velocity, but all disturbances of zero group velocity are damped. Thus, the amplified wave packets grow as they are convected out of the domain. These flows tend to

(a)

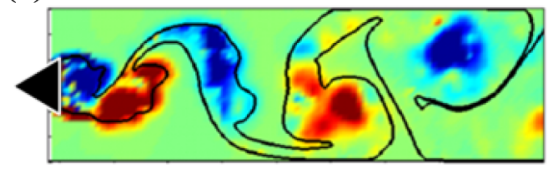

(b)

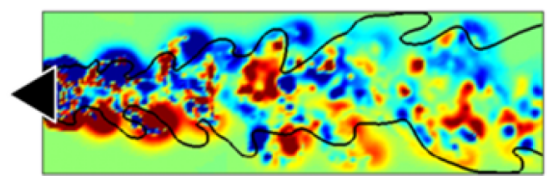

(c)

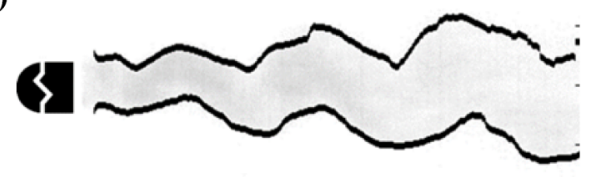

(d)

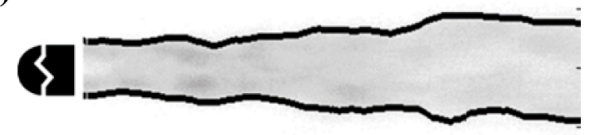

FIG. 1. Left column: computationally predicted vorticity field and instantaneous flame edge for two flame density ratios, (a) $\rho_{u} / \rho_{b}=1.25$ and (b) $\rho_{u} / \rho_{b}=2.0$, reproduced with permission from R. R. Erickson and M. C. Soteriou, "The influence of reactant temperature on the dynamics of bluff body stabilized premixed flames," Combust. Flame 158, 2441 (2011). Copyright 2011 Elsevier. Right column: chemiluminescence flame images at two density ratios, (c) $\rho_{u} / \rho_{b}=1.7$ and (d) $\rho_{u} / \rho_{b}=3.2$, from the authors' experimental work. ${ }^{20}$ 
be highly receptive to excitation and behave as disturbance amplifiers. ${ }^{22-24}$ Globally unstable flows and absolutely unstable flow profiles are self-excited, meaning that they continue to oscillate in the lab-fixed reference frame once they are perturbed. Unlike a convectively unstable flow, oscillations persist without continuous excitation. Absolute instability is a local concept, meaning that it describes the stability of local flow profiles, typically at a fixed axial position. In real flows where the mean flow profile develops axially, a sufficiently large "pocket" of absolute instability can serve as the wave-maker which is the source of global instability, whose spatial pattern is known as the global mode. ${ }^{22-24}$ Thus, flows containing regions with large, positive absolute growth rates tend to be globally unstable. In the case of the bluff body wake, the global instability manifests itself as the sinuous vortical structures discussed previously. ${ }^{12}$

$\mathrm{Yu}$ and Monkewitz ${ }^{25}$ performed a local stability analysis of a model wake flow with non-uniform density. They parameterized the flow with two stability parameters: the density ratio, $S=\rho_{b} / \rho_{u}$, and a shear ratio, $\lambda=\left(U_{b}-U_{u}\right) /\left(U_{b}+U_{u}\right)$, where the base density, $\rho$, and base velocity, $U$, are marked with a subscript $b$ in the wake region and are marked with a subscript $u$ in the free-stream region. For the reacting wake problem, $\lambda<0$ and $S<1$. They showed that flows with a high level of reverse flow, which corresponds to a high $|\lambda|$, are absolutely unstable. Additionally, they showed that flows with $S$ close to 1 (small density jumps) are absolutely unstable. In addition, Emerson et al. ${ }^{20,26}$ showed that the spatial offset between the time averaged density and velocity points of inflection also had strong influences on the global stability of the reacting wake. As such, the spreading of the flame (controlled by the turbulent flame speed) relative to the shear layer has significant influences on the flame density ratio values at which self-sustained oscillations occur. These model stability analyses predict two important qualitative trends - absolute instability growth rates of a wake flow increase as density ratio decreases, and as backflow ratio increases.

The majority of existing analyses and experiments have focused on symmetric configurations (symmetric geometry and time-averaged flow). ${ }^{20,21,27,28}$ A notable exception is the recent experiments by Tuttle et al. ${ }^{29}$ who analyzed the dynamics of combusting wakes which were fueled with varying degrees of transverse stratification with respect to the bluff body centerline. Figure 2(a) shows their stratified equivalence ratio profile, where the nominal equivalence ratio, $\phi$, varies between $\phi-\phi_{\varepsilon}$ and $\phi+\phi_{\varepsilon}$ at $y=H$ and $y=-H$, respectively. Figure 2(b) shows a typical chemiluminescence image of a flame with stratified equivalence ratio, obtained from this facility. Their data show that a narrowband feature in the chemiluminescence spectrum appears and increases in amplitude with increases in level of equivalence ratio stratification. ${ }^{29}$ Interpreted in the light of the above hydrodynamic stability discussion, this result suggests that wake density asymmetry promotes global instability. The objective of this study is to parameterize base flow asymmetry parameters upon the hydrodynamic stability trends of the flow. The model is also compared to experimental data of Tuttle et al. ${ }^{29}$ to assess its ability to capture the key qualitative effects of these base flow asymmetry parameters.

\section{STABILITY ANALYSIS FORMULATION}

This section describes the stability analysis formulation, with greater details shown in the Appendix. The base flow whose stability is analyzed here consists of a recirculating wake with density

(a)

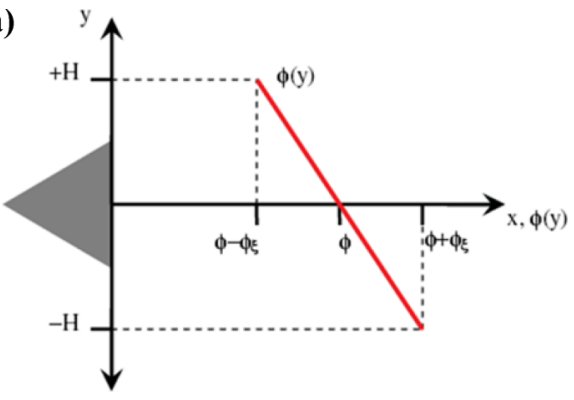

(b)

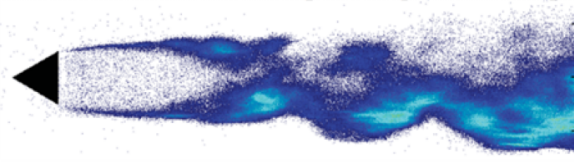

FIG. 2. (a) Equivalence ratio profile and (b) chemiluminescence image of a flame with stratified equivalence ratio. Reproduced with permission from Tuttle et al., "Lean blowoff behavior of asymmetrically-fueled bluff body-stabilized flames," Combust. Flame 160, 1677 (2013). Copyright 2013 Elsevier. 


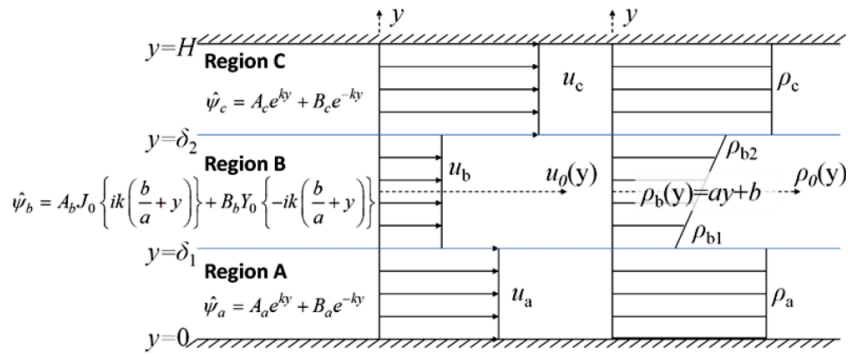

FIG. 3. Model wake base flow with density stratification and asymmetry in the base velocity and density.

stratification and confinement. The base flow, shown in Figure 3, is modeled as three regions, which contain smooth, continuous velocity and density profiles, separated by discontinuous jumps. These three regions represent different physical parts of the flow. The outer regions, denoted (a) and (c), represent the outer reactant flow. The inner region, denoted (b), represents the recirculating wake of combustion products. The interfaces between these regions consist of jump discontinuities, located at $y=\delta_{1}$ and $y=\delta_{2}$, which represent the flames and shear layers. The base velocity profile is formulated to allow asymmetry, by permitting different outer velocities on either sides of the bluff body. The base density profile also includes asymmetry, by modeling a constant outer (reactant) density and by allowing density jumps of differing magnitudes on either sides of the bluff body. These density jumps physically represent the density drops across flames of different exothermicities, for example, due to a stratified equivalence ratio.

The velocity jump discontinuities physically represent vorticity sheets and serve as unsteady vorticity sources. ${ }^{26}$ These unsteady vorticity sources play a key role in the hydrodynamic stability of this base profile. We define inner, outer, and average velocities as

$$
\begin{aligned}
U_{\text {inner }} & =U_{b}, \\
U_{\text {outer }} & =\frac{1}{2}\left(U_{a}+U_{c}\right)=U_{\text {lip }}, \\
U_{a v} & =\frac{1}{2}\left(U_{\text {outer }}+U_{\text {inner }}\right) .
\end{aligned}
$$

Thus, $U_{\text {outer }}$ denotes the average velocity of the reactant streams (the bluff body lip velocity) and $U_{a v}$ denotes the overall average velocity at a given axial station. We define a bulk shear parameter, $\lambda$, and a velocity asymmetry parameter $\chi_{u}$ as

$$
\begin{aligned}
\lambda & =\frac{U_{\text {inner }}-U_{\text {outer }}}{U_{a v}}, \\
\chi_{u} & =\frac{U_{c}-U_{a}}{U_{a v}} .
\end{aligned}
$$

Similarly, we define an outer (un-burned) density, a linearly varying inner (burned) density with density extremes of $\rho_{b 1}$ and $\rho_{b 2}$, and an average density

$$
\begin{aligned}
\rho_{\text {outer }} & =\rho_{a}=\rho_{c}, \\
\rho_{\text {inner }} & =\frac{1}{2}\left(\rho_{b 1}+\rho_{b 2}\right), \\
\rho_{\text {av }} & =\frac{1}{2}\left(\rho_{\text {outer }}+\rho_{\text {inner }}\right) .
\end{aligned}
$$

From these densities, we form a bulk density ratio, $S$, and a density asymmetry parameter, $\chi_{\rho}$,

$$
\begin{aligned}
S & =\frac{\rho_{\text {inner }}}{\rho_{\text {outer }}}, \\
\chi_{\rho} & =\frac{\rho_{\text {b2 }}-\rho_{\text {b1 }}}{\rho_{\text {av }}} .
\end{aligned}
$$




\section{STABILITY ANALYSIS RESULT}

Section II above details a stability analysis with five parameters: density ratio, $S$, shear ratio, $\lambda$, density asymmetry, $\chi_{\rho}$, velocity asymmetry, $\chi_{u}$, and a confinement ratio, $\beta=H / D$. For the results shown in this section, the confinement ratio was held constant at $\beta=4$, the value corresponding to that used in the experiments considered later. Previous stability analyses have elucidated the influence that $S$ and $\lambda$ have on the flow stability. ${ }^{25,30}$ As such, this section focuses on the influence of the asymmetry parameters on the flow stability, which may be compared to the experimental results of Tuttle et al. ${ }^{29}$ It is important to note that this section presents quantitative stability predictions (frequencies, growth rates, critical parameters for absolute/convective transition, etc.) in the context of the base flow model, which describes a reduced set of stability parameters. When these stability predictions are later compared to the experiment, they are not meant as quantitative predictions of the experiment but instead are meant to indicate the sensitivities and directional trends that the modeled parameters have in the experiment.

The traditional bluff body wake problem has multiple absolute instability modes: some which are sinuous and some which are varicose. As the degree of base flow asymmetry is increased, these modes distort from the canonical sinuous and varicose structures. In this section, we focus on the most absolutely unstable mode, which is a distortion of the sinuous mode over the studied parameter space. In other words, this mode always belongs to a solution branch which reverts to the classical sinuous mode when base flow symmetry is restored, i.e., when $\chi_{u}=\chi_{\rho}=0$. Figure 4 shows the influence of $\chi_{\rho}$ on the most absolutely unstable hydrodynamic pressure mode shape for a density ratio of $S=0.7$, a fixed shear ratio of $\lambda=-2$, and no velocity asymmetry $\left(\chi_{u}=0\right)$. The figure shows the distortion of the nominally sinuous mode, induced by density assymetry-in particular, it shows that relatively larger pressure fluctuations occur on the side with the smaller density jump. Although the mode shapes of the hydrodynamic velocity are not shown, the axial and transverse velocity components also have greatest magnitude on the side with the smaller density jump, as quantified next for the transverse velocity. Note that this is a linear analysis; thus, the transverse dependence of a given mode's magnitudes is meaningful, but the relative scaling of two different modes is arbitrary. Therefore, these mode shapes are normalized to facilitate comparison, and the relative magnitudes of the $\chi_{\rho}=0$ and $\chi_{\rho}=1.1$ modes should not be compared.

The magnitude and phase of the unsteady velocity component normal to the flame have a major influence on the magnitude of flapping of bluff body flames. ${ }^{28}$ For nearly flat flames, like those encountered in most high speed flows, the transverse velocity component is roughly equal to the flame-normal velocity. Therefore, Figure 5(a) plots the ratio of the transverse velocity magnitude at the smaller density jump (denoted $\left|\hat{v}_{L}\right|=\left|\hat{v}\left(y=\delta_{2}\right)\right|$ ), relative to the transverse velocity magnitude

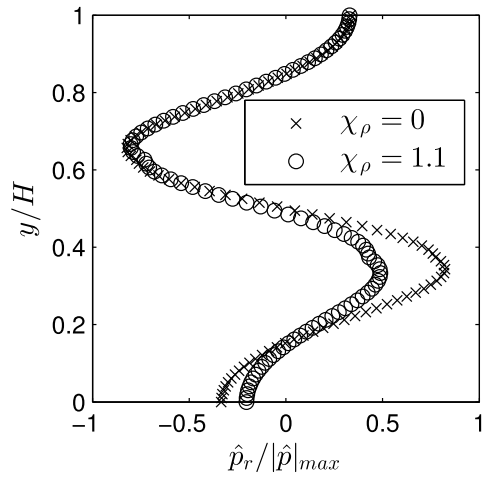

(a)

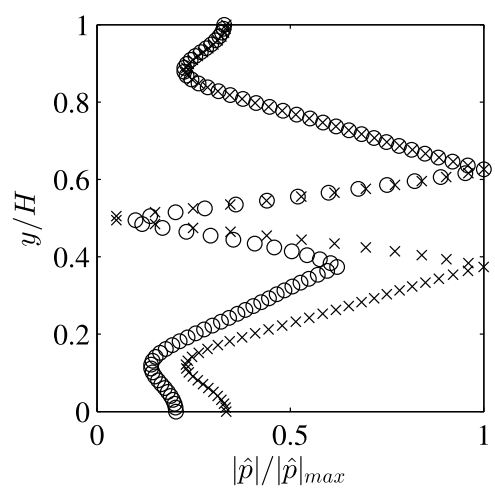

(b)

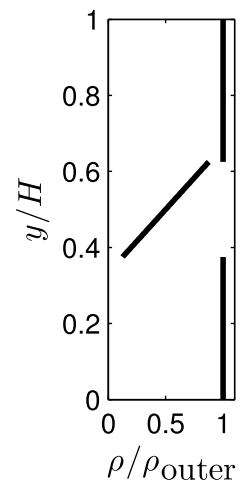

(c)

FIG. 4. Influence of density asymmetry on the most absolutely unstable mode shape. Results are shown for the hydrodynamic pressure, for two different levels of base flow density asymmetry and $S=0.7$. Part (a) shows a snapshot of the mode shape (the real part), and part (b) shows the magnitude of the pressure mode. In (a), the two modes are displayed so that their phases at $y / H=1$ are the same. Part (c) shows stratified density profile. 


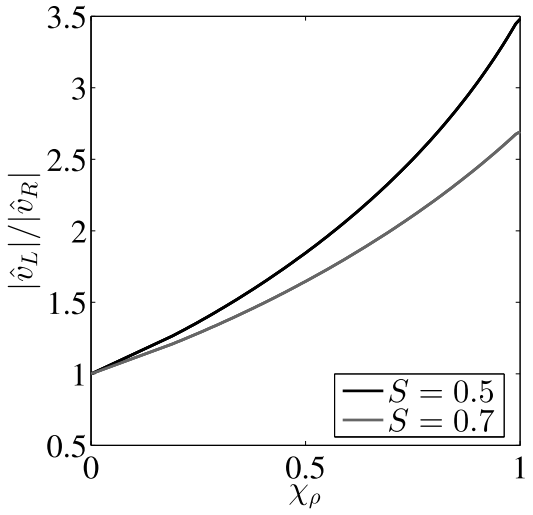

(a)

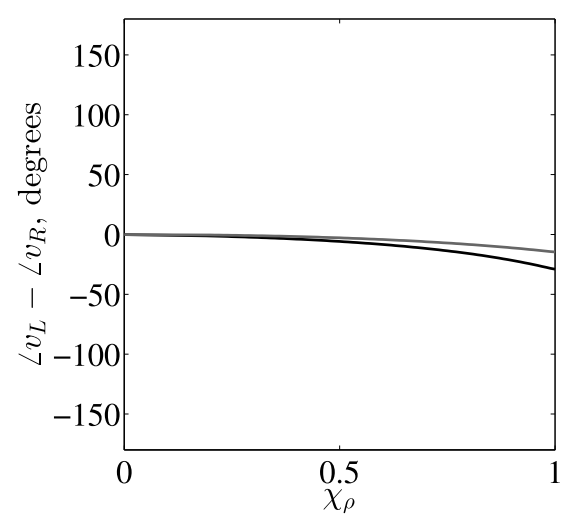

(b)

FIG. 5. Comparison of transverse velocity fluctuations at the two density jumps, showing (a) ratio of unsteady transverse velocity magnitude and (b) phase difference between unsteady transverse velocities at the smaller density jump (at $\left.y=\delta_{2}\right)$ and the bigger density jump (at $y=\delta_{1}$ ), for $\chi_{\rho}=1$ and $\chi_{u}=0$.

at the bigger density jump (denoted $\left.\left|\hat{v}_{R}\right|=\left|\hat{v}\left(y=\delta_{1}\right)\right|\right)$. The figure demonstrates that $\chi_{\rho}$ has a strong influence on this measure of transverse unsteady velocity asymmetry. For example, at $\chi_{\rho}=1$ and $S=0.5$, the magnitude of transverse velocity fluctuation at the smaller density jump is 3.5 times greater than that at the bigger density jump, such that we expect much stronger flapping of the leaner flame branch relative to its neighboring richer flame branch. Figure 5(b) shows the phase difference between the transverse velocity fluctuations at each of the two density jumps. This plot demonstrates that the phase difference between the oscillatory velocities at the two flame branches is relatively insensitive to base density asymmetry. For example, at $S=0.5$, the phase difference approaches $30^{\circ}$ at high values of $\chi_{\rho}$, compared to a phase difference of $0^{\circ}$ for a symmetric base flow. This has implications on the combusting wake application, as it suggests that base density stratification would not strongly influence the relative phasing of the two flame branches.

Figure 6 shows the influence of base flow density asymmetry, $\chi_{\rho}$, on the absolute growth rate and frequency. Results are shown for two fixed density ratios, $S$, and no base flow velocity asymmetry, $\chi_{u}=0$. Figure 6(a) shows that increasing $\chi_{\rho}$ is destabilizing. For example, a symmetrically stratified wake with a density ratio of $S=0.5$ is absolutely stable (and convectively unstable), but increasing $\chi_{\rho}$ to a value of roughly 0.6 causes a transition from convective instability to absolute instability. This critical value of $\chi_{\rho}$ is a function of $S$ and $\lambda$ and is denoted as $\chi_{\rho}{ }^{*}(S, \lambda)$. Figure $6(\mathrm{~b})$ shows the

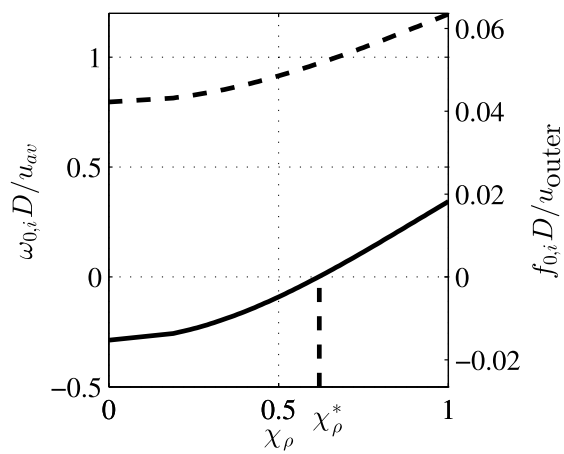

(a)

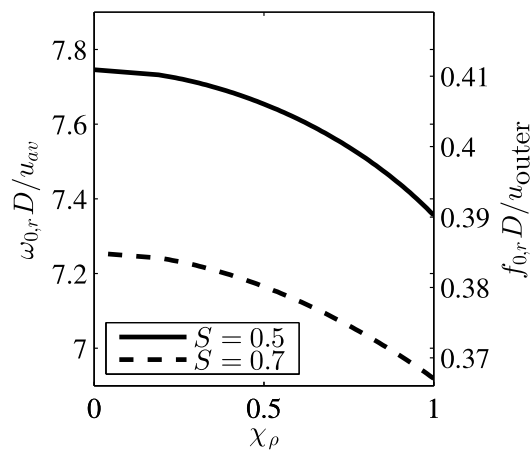

(b)

FIG. 6. Influence of base flow density asymmetry on the absolute (a) growth rate and (b) frequency, for two different density ratios and $\lambda=-2$. Part (a) graphically defines the critical level of density stratification, $\chi_{\rho}{ }^{*}(S)$. Two ordinate axes are shown on each plot: the left axis shows the natural hydrodynamic scaling, and the right axis shows the classical empirical Strouhal scaling. 


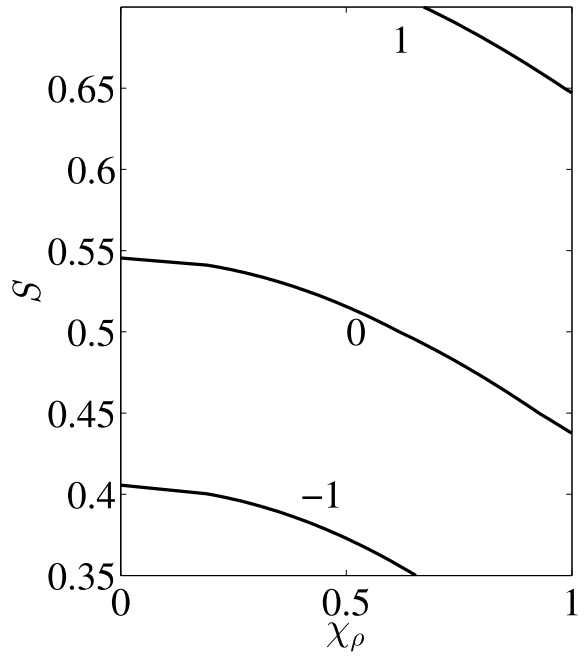

(a)

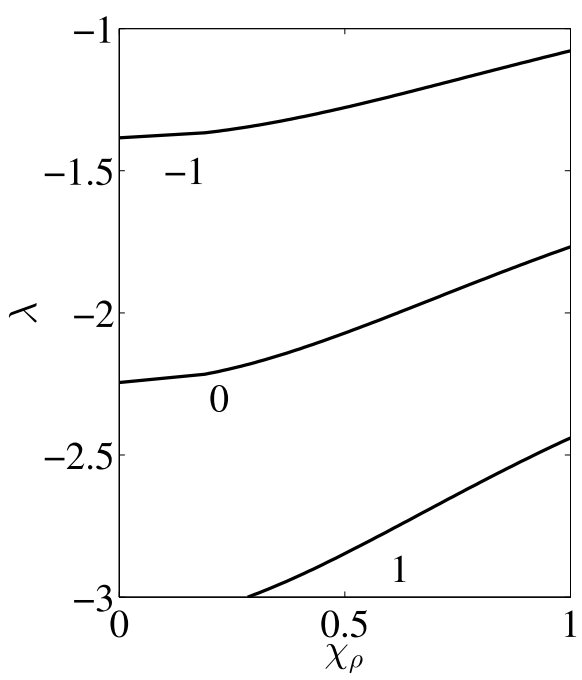

(c)

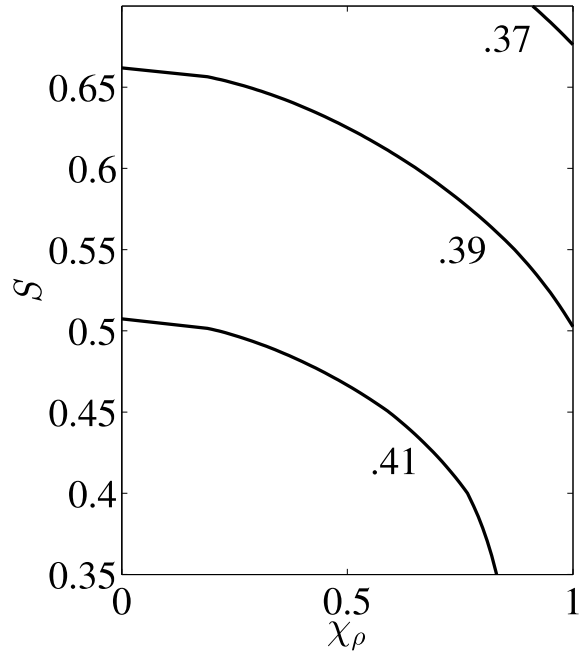

(b)

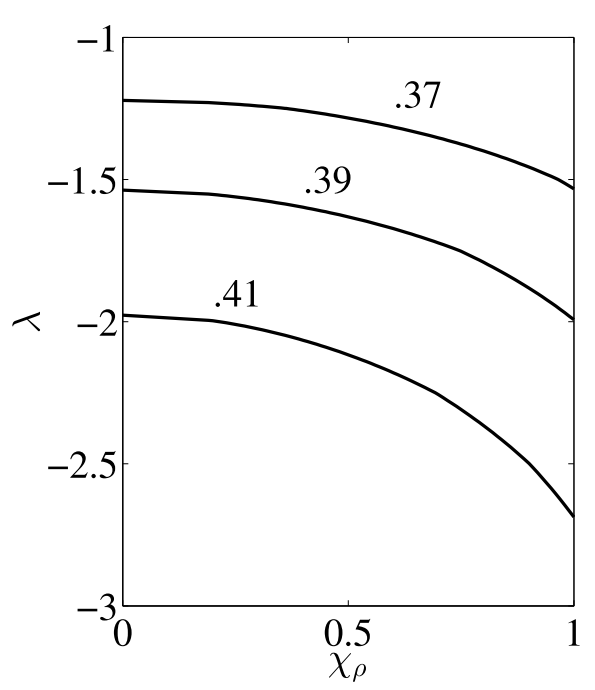

(d)

FIG. 7. Contours of absolute growth rate, $\omega_{0, i} D / u_{a v}$, as a function of the base flow density asymmetry and (a) the density ratio with $\lambda=-2$, (c) the shear ratio with $S=0.5$. Absolute frequency contours, $f_{0, r} D / u_{\text {outer }}$, are plotted in (b) and (d) for same conditions as parts (a) and (c), respectively. The $\omega_{0, i} D / u_{a v}=0$ contour in (a) and (c) indicates the stability limit, $\chi_{\rho}{ }^{*}(S, \lambda)$.

influence of $\chi_{\rho}$ on the absolute frequency. An increase in $\chi_{\rho}$ generally reduces the absolute frequency. However, the sensitivity of the absolute frequency to $\chi_{\rho}$ is relatively low over the range of $\chi_{\rho}$ that is physically relevant. The range of physically relevant values of $\chi_{\rho}$ is discussed later. The influence of $\chi_{\rho}$ on the absolutely unstable mode is likely rooted in the baroclinic mechanism, which is the dominant physical mechanism through which the density jumps affect the stability of this flow. ${ }^{26}$ Figure 6 uses two ordinate axes- one labeled as $\omega D / u_{a v}$ and one labeled as $f_{0, r} D / u_{\text {outer }}$, where $f_{0, r}=\omega_{0, r} / 2 \pi$. We do this to demonstrate the relative values of these two classical frequency scalings, the first of which is the natural nondimensionalization that occurs in model stability analyses, and the second which is the classical empirical Strouhal scaling.

As noted above, the critical level of base flow density asymmetry, $\chi_{\rho}{ }^{*}$, is a function of $S$ and $\lambda$. This interdependence is shown in Figure 7(a), which plots the absolute growth rate as a function of $\chi_{\rho}$ and $S$ for a given shear parameter, $\lambda$, and as a function of $\chi_{\rho}$ and $\lambda$ for a fixed $S$. For example, 


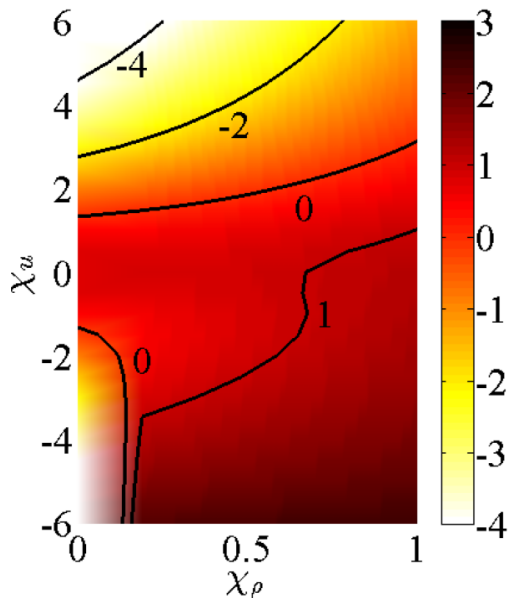

(a)

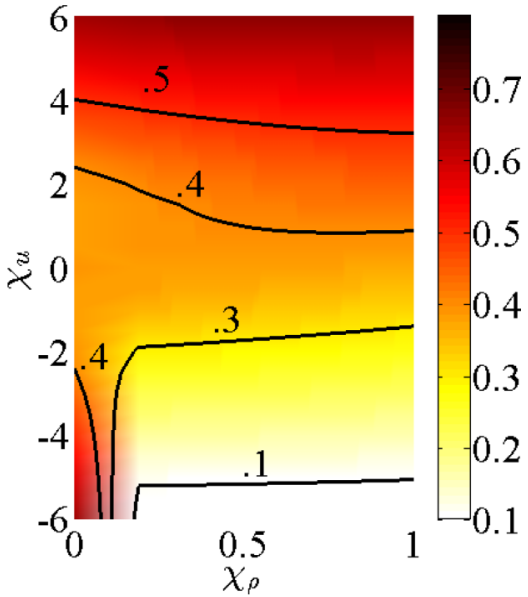

(b)

FIG. 8. Influence of base flow density and velocity asymmetry on the absolute (a) growth rate, $\omega_{0, i} D / u_{a v}$, and (b) frequency, $f_{0, r} D / u_{\text {outer }}$, for $S=0.7$ and $\lambda=-2$.

in the absence of base flow density asymmetry, and with a shear ratio of $\lambda=-2$, the critical density ratio is roughly $S=0.55$. However, when $\chi_{\rho}=1.0$, the critical density ratio drops to $S=0.45$.

To put some specific numbers to these calculation, assuming a reactant temperature of $1100 \mathrm{~K}$, with a $2100 \mathrm{~K}$ flame on one side and $2600 \mathrm{~K}$ flame on the other (temperatures roughly corresponding to upper and lower reactant streams of $\phi=0.5$ and 1.0, respectively), then $S=0.5$ and $\chi_{\rho}=0.15$. This flow is absolutely stable. If the leaner flame were to extinguish and its "burned" temperature were to revert to that of the approach flow, then $S=0.7$ and $\chi_{\rho}=0.7$. This flow would be absolutely unstable. This example shows the potential feedback between flame blow off and hydrodynamic stability that has been hypothesized by a number of workers. ${ }^{1,31}$ A value of $\chi_{\rho}=1.0$ could be achieved with $500 \mathrm{~K}$ reactants, a $2300 \mathrm{~K}$ flame branch on one side, and an extinguished branch on the other.

We next consider the effects of base flow velocity asymmetry. Figure 8 shows the absolute stability of the wake as a function of base flow velocity asymmetry, $\chi_{u}$, and base flow density asymmetry, $\chi_{\rho}$. The figure shows a color bar in addition to iso-contours to help elucidate the somewhat complicated topology of the data. The figure shows that increases in the degree of base flow velocity asymmetry have a stabilizing effect on the absolute instability mode. This result makes intuitive sense, since increasing the base velocity asymmetry shapes the base flow to look less like a wake, and more like a simple shear layer (which is convectively unstable for this value of forward to reverse flow velocity). Interestingly, the stabilizing influence of the base velocity asymmetry is more sensitive when $\chi_{u}>0$ and $\chi_{\rho}>0$, which for this arrangement puts the stronger shear layer near the smaller density jump. This makes physical sense from a group velocity point of view, since this makes the faster forward flow the denser flow and the reversing or slower forward flow the less dense flow. This high downstream momentum congifiguration may be reasoned to promote non-zero group velocity, and thus absolute stability.

\section{EXPERIMENTAL DATA}

This section presents experimental data obtained by Tuttle et al. ${ }^{29}$ which was described in Section I. The end of this section compares the available experimental results to the stability analysis to demonstrate some of the predicted parametric dependencies. Results were obtained for three experimental conditions, summarized in Table I. In the table, three equivalence ratios are shown: the equivalence ratio at the flow centerline, $\phi$, and the equivalence ratios estimated at the leaner and richer flame branches, $\phi_{L}$ and $\phi_{R}$, respectively. In this combustion scenario, the leaner flame branch generates a smaller density jump, and the richer flame branch generates a bigger density jump. The values of $\phi_{L}$ 
TABLE I. Experimental conditions, including estimated equivalence ratios at each flame branch.

\begin{tabular}{lccccc}
\hline \hline$G$ & $\phi$ & $\phi_{R}$ & $\phi_{L}$ & $S$ & $\chi_{\rho}$ \\
\hline 0.0 & 0.51 & 0.51 & 0.51 & 0.2 & 0.0 \\
0.5 & 0.45 & 0.70 & 0.20 & 0.3 & 0.3 \\
1.0 & 0.27 & 0.77 & 0.0 & 0.6 & 1.1 \\
\hline \hline
\end{tabular}

and $\phi_{R}$ are estimated from the equivalence ratio gradients, $G$, reported in the work of Tuttle et al. ${ }^{29}$ Next, the values of $S$ and $\chi_{\rho}$ are estimated from an equilibrium calculation using the equivalence ratios, $\phi_{L}$ and $\phi_{R}$, and the preheat temperature reported in the work of Tuttle et al. ${ }^{29}$ We emphasize the fact that $S$ and $\chi_{\rho}$ are simple parameters that are calculated from the data; they serve to indicate the relative levels of density stratification and asymmetry between the three cases.

The experimental diagnostics used for this comparison, detailed in the work of Tuttle et al., ${ }^{29}$ include low speed Particle Image Velocimetry (PIV) and $6 \mathrm{kHz}$ chemiluminescence imaging. The fact that only low speed PIV data are available precludes quantitative comparisons of the stability theory and measured flow field but does allow us to fit the model to the time averaged velocity data to estimate the stability parameters. For example, Figure 9(a) shows a slice of the measured time-averaged axial velocity at $x / D=1$. In order to determine a representative value of the $U_{\text {outer }}$ and $U_{\text {inner }}$ parameters associated with this operating condition on the stability plot shown later in Figure 10, Figure 9 also shows the fit of the measured data to the model profile presented earlier. The outer velocity was

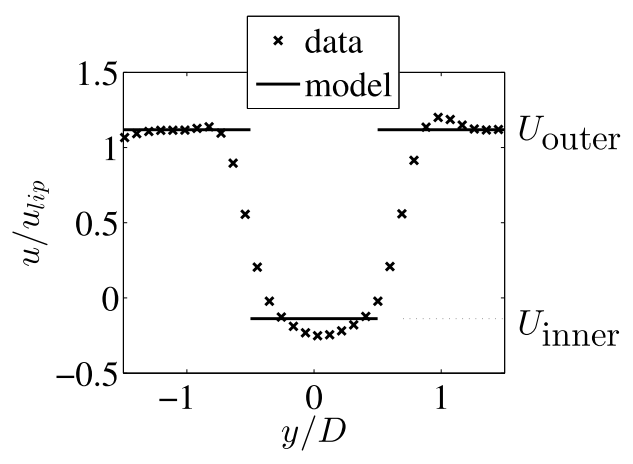

(a)

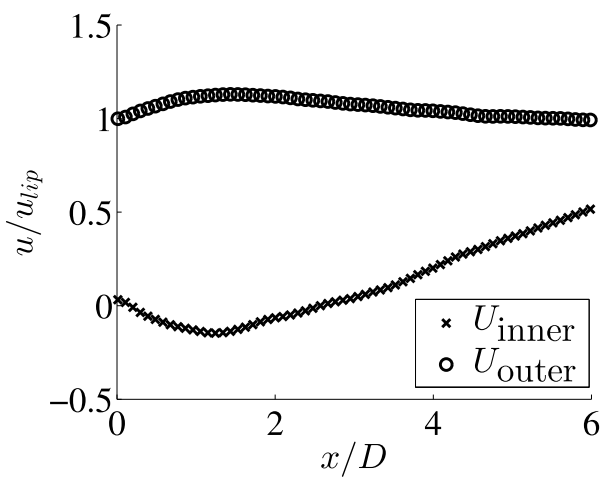

(b)

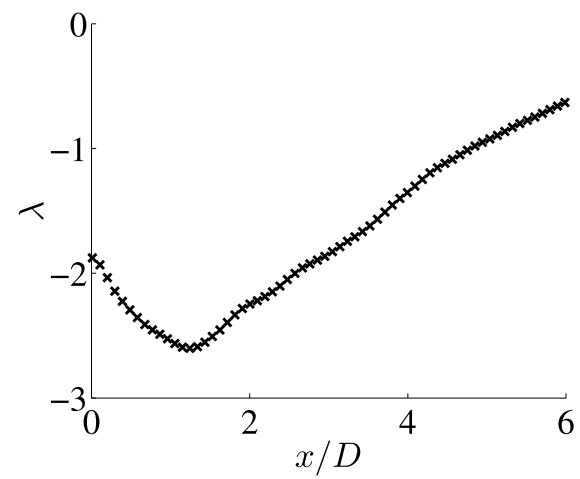

(c)

FIG. 9. Measured time averaged velocity field, showing (a) example overlay of model on velocity data at $x / D=1$, (b) axial dependence of the $U_{\text {outer }}$ and $U_{\text {inner }}$ fits, and (c) the resulting axial dependence of $\lambda$. Plots are shown for the $\chi_{\rho}=0$ case but are representative of all cases. 


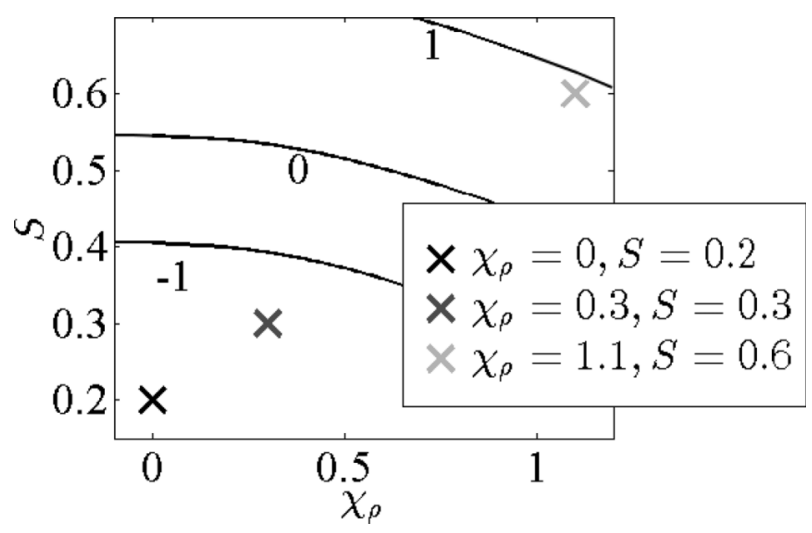

FIG. 10. "Zoomed out" view of the stability map from Figure 7(a), showing contours of absolute growth rate, $\omega_{0, i} D / u_{a v}$ Estimated locations of experimental conditions (see Table I) are marked with "x."

calculated as the average axial velocity in the region of $1<|y / D|<1.5$, and the inner velocity was estimated as the average axial velocity in the region $|y / D|<0.5$. Figures 9 (b) and 9(c) show the axial dependence of the $U_{\text {outer }}$ and $U_{\text {inner }}$, and $\lambda$, which result from this procedure. The figure demonstrates that $\lambda$ is a function of axial position, with values on the order of $\lambda=-2$ for the first two bluff body diameters. This highlights the importance of gleaning qualitative parametric dependencies from the local stability analysis, rather than quantitative predictions of the absolute/convective stability transition. For all cases considered in this paper, the bluff body diameter was $D=9.6 \mathrm{~mm}$, and the lip velocity was $u_{\text {lip }}=18.4 \mathrm{~m} / \mathrm{s}$.

The three cases listed in Table I are marked on a stability map as shown in Figure 10. The figure is parameterized in terms of the density ratio $S$ and the degree of base density asymmetry $\chi_{\rho}$. Figure 10 also indicates contours of absolute growth rate, also plotted in Figure 7, but over a broader parameter space that covers the range of values of $S$ and $\chi_{\rho}$ that are investigated with this data set. Note that the stability parameters estimated for the three experimental cases traverse the stability map across a broad range of absolute growth rates.

We extracted flame edges from high speed chemiluminescence images to quantify the space-time dynamics of the two flame branches independently, using the same procedure as detailed in Emerson et $a .^{20}$ The spatio-temporal evolution of the flame edge is quantified by the variable $L(x, t)$. The fluctuating flame position, $L^{\prime}(x, t)$, is obtained by subtracting the time averaged value of $L(x, t)$, as illustrated in Figure 11(b). Figure 11 shows examples of flames at two different levels of $\chi_{\rho}$, with the extracted edges overlaid. Note that in Figure 11(b), coherent, large scale wrinkling is observed in the lean flame branch (the top, emboldened one), but the flame is much smoother and incoherently wrinkled on the lower, rich flame. The appearance of this coherent wrinkling is highly intermittent, similar to other experimental investigations of global mode oscillations of turbulent bluff body wakes. ${ }^{20}$

In order to better quantify the flame edge dynamics, we Fourier transform the flame edge displacement, $L^{\prime}(x, t)$. The Fourier transformed flame edge displacement is denoted $\left|\hat{L}_{L}(x, S t)\right|$ for the leaner flame branch and $\left|\hat{L}_{R}(x, S t)\right|$ for the richer flame branch. Here, St is the Strouhal number, defined as $S t=f D / u_{\text {lip }}$, where $D$ is the bluff body diameter and $u_{\text {lip }}$ is the bluff body lip velocity. Figure 12 shows typical spectra of flame edge displacement oscillations for cases with and without base density asymmetry. The figure shows a prominent narrowband feature near $S t=0.11$. We speculate that at this frequency, the flame is wrinkling as the result of vortically induced motions that are excited by background longitudinal acoustics. As evidence, the two flame branches move $180^{\circ}$ out of phase with each other at this frequency, indicating a varicose flow response like that typical of longitudinally acoustically forced wakes. ${ }^{28}$ Therefore, we focus the analysis on a different spectral band where the global wake mode is classically observed ${ }^{32}$ and predicted in the analyses shown in Figures 6 and 7 , i.e., in the range of $0.2<S t<0.5$.

In order to capture the dynamics associated with the global mode, we integrate the spectral energy of $\hat{L}^{\prime}$ in the range of $0.2<S t<0.5$, indicated by the gray shading in Figure 12. Following the 


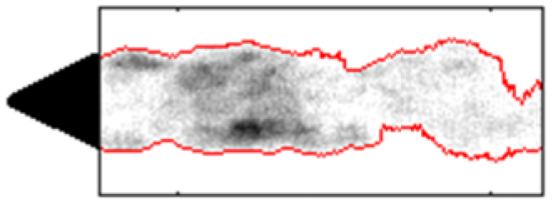

$\mathrm{t}=0 \mathrm{~ms}$

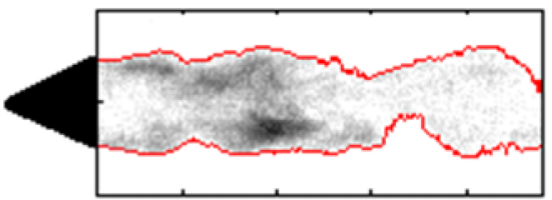

$\mathrm{t}=0.167 \mathrm{~ms}$

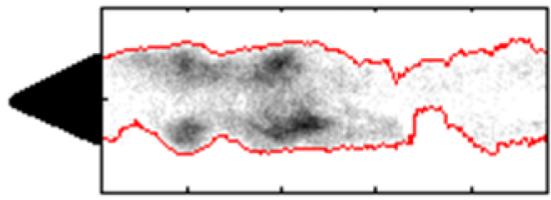

$\mathrm{t}=0.333 \mathrm{~ms}$

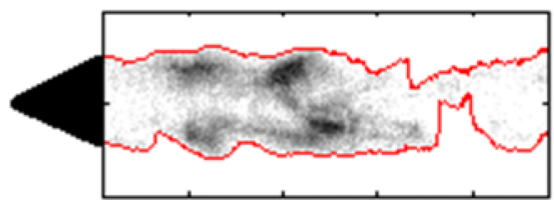

$\mathrm{t}=0.5 \mathrm{~ms}$

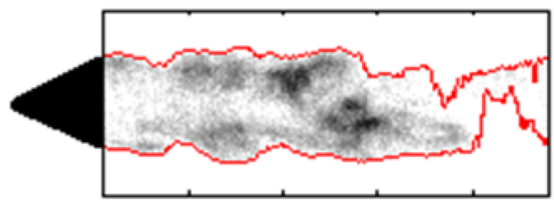

$\mathrm{t}=0.667 \mathrm{~ms}$

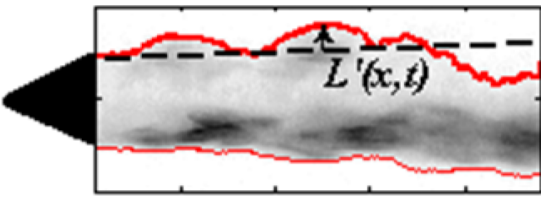

$\mathrm{t}=0 \mathrm{~ms}$

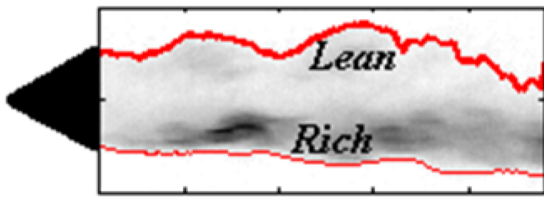

$\mathrm{t}=0.167 \mathrm{~ms}$
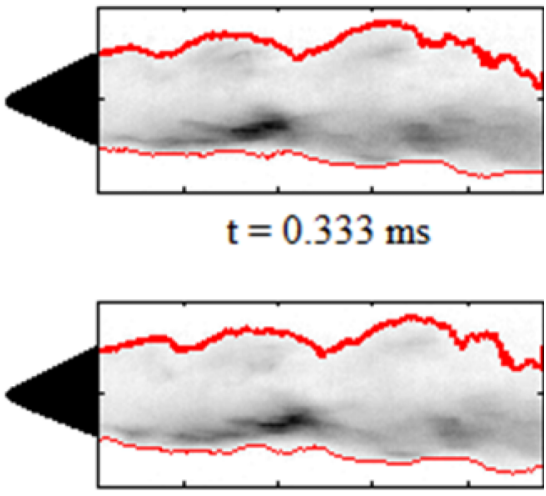

$\mathrm{t}=0.5 \mathrm{~ms}$

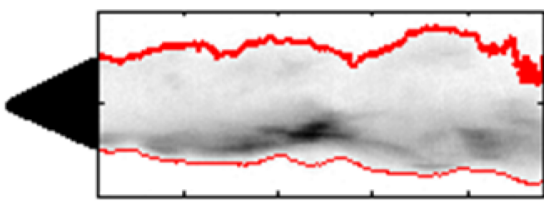

$\mathrm{t}=0.667 \mathrm{~ms}$

(a)

(b)

FIG. 11. Series of chemiluminescence flame images for (a) symmetric density stratification remove bold line from part (a), $\chi_{\rho}=0$ and (b) asymmetric density stratification, $\chi_{\rho}=1$.1. Flame edge detection is overlaid for both flame edges. The flame edge with smaller density jump (upper flame edge) is emboldened in all frames of column (b) and is labeled in the second frame of column (b).

procedure outlined in Emerson et al. ${ }^{20}$ we use Parseval's theorem to express this integrated spectral energy as a root mean square fluctuation, denoted as $L_{r m s}^{\prime}$. Figure 13 compares the axial dependence of $L_{r m s}^{\prime}(x)$ for the leaner and richer flame branches (denoted $L_{L, r m s}^{\prime}$ and $L_{R, r m s}^{\prime}$, respectively), for all three values of $\chi_{\rho}$. The figure makes this comparison in two ways: by considering the average value of $L_{r m s}^{\prime}$ between the two flame branches and by comparing the ratio of $L_{r m s}^{\prime}$ of one branch to that of the other.

The results in Figure 13 show the influence of density asymmetry predicted by the stability analysis. The influence of $\chi_{\rho}$ is highly evident when considering the relative $L_{r m s}^{\prime}$ of the two flame branches. For example, the low $\chi_{\rho}$ cases exhibit equal values of $L_{r m s}^{\prime}$ for the two flame branches. In contrast, the high $\chi_{\rho}$ case shows quite different flame edge oscillation amplitudes, differing by a factor of 3 for the leaner flame edge than for the richer flame edge. Referring to Figure 5, the corresponding 


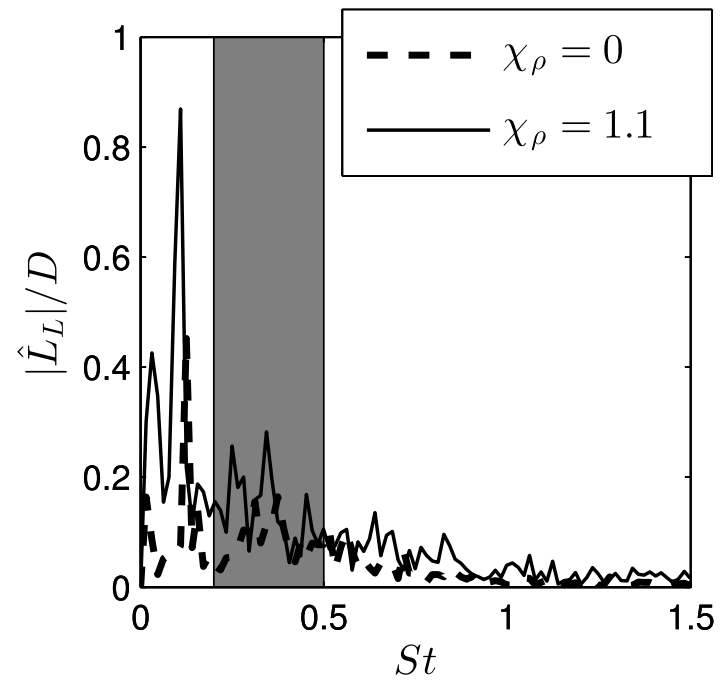

FIG. 12. Ensemble averaged spectra of the leaner (lower density jump) flame edge displacement fluctuations for two values of $\chi_{\rho}$. Gray shading indicates Strouhal number region spanning $0.2<S t<0.5$.

value of transverse velocity asymmetry predicted by the stability analysis is roughly 3 . Moreover, the flame branch with the largest oscillations is the leaner one (with the smaller density jump), which is where the analysis showed that the largest transverse velocity fluctuations occur. This result supports the discussion surrounding in Figure 4, which predicts that at high $\chi_{\rho}$, the global mode oscillations should be higher on the side of the flow with the leaner flame than on the side with the richer flame. Interestingly, $\chi_{\rho}$ does not exhibit a significant impact on the value of $L_{r m s}^{\prime}$ averaged between the two flame branches for cases with based density asymmetry. In other words, the augmented flapping of the leaner branch is counteracted by diminished flapping of the richer branch.

The key finding of the comparison between experiment and model is that both clearly demonstrate that the hydrodynamic oscillations should have greater magnitude at the leaner flame branch than at the neighboring richer flame branch. This finding has interesting analogies to the symmetric density profile case, which shows that absolute instability growth rates increase with decreasing density ratio. ${ }^{25}$ For the case of symmetric base flow, the stabilizing effect of increased density ratio

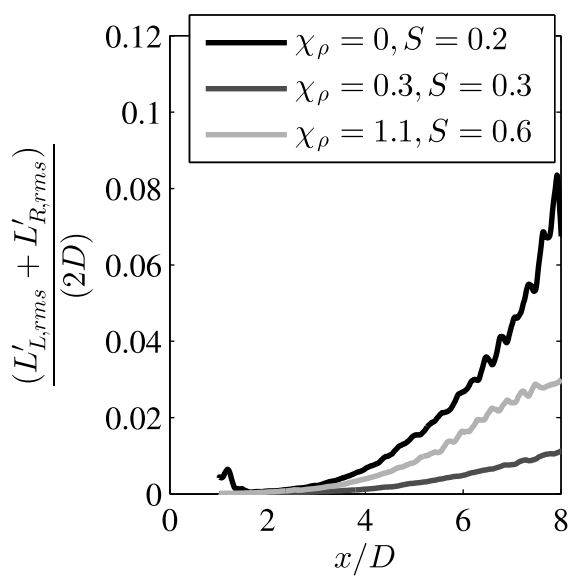

(a)

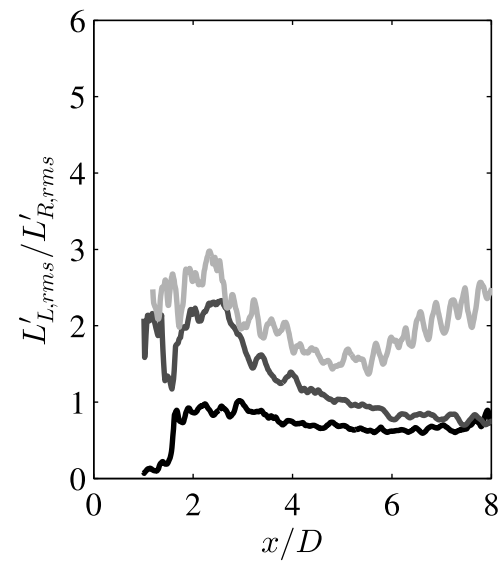

(b)

FIG. 13. (a) Axial dependence of $L_{r m s}^{\prime}$ averaged for the two flame branches. (b) Ratio of $L_{r m s}^{\prime}$ for the leaner flame branch to that of the richer flame branch. 
is associated with baroclinic torque. The baroclinic torque has been shown to be stabilizing ${ }^{32}$ since its oscillatory component tends to act out of phase with the other hydrodynamic mechanisms in the wake. ${ }^{26}$ We also note the observation that the reversing flow, which is often associated with absolute instability, has higher density on the side of the flow with the leaner flame. Therefore, the side of the flow with the greater reverse flow momentum exhibits the larger hydrodynamic oscillations.

\section{CONCLUSIONS}

Hydrodynamic stability analyses of reacting bluff body wakes have parameterized the reacting wake problem in terms of the density ratio and backflow ratio, ${ }^{25}$ the confinement, ${ }^{33}$ and the spatial offset between the flame and the shear layer. ${ }^{20} \mathrm{~A}$ recent series of experiments performed by Tuttle et al. ${ }^{29}$ has shown that base flow asymmetry is also an important stability parameter. In these experiments, the bluff body was fueled with a spatially stratified equivalence ratio such that the density field was asymmetric.

This paper extends prior stability analyses to include the effects of base flow asymmetry. The stability analysis predicts that increasing the degree of base flow density asymmetry has a destabilizing effect. Additionally, the stability analysis indicates that increasing the degree of density asymmetry distorts the classical sinuous mode, and in such a way that the side of the flow with the smaller density jump exhibits larger pressure and velocity fluctuations. These results are compared to the experimental results from Tuttle et al. ${ }^{29}$ This comparison supports the stability analysis and shows that at high levels of base density asymmetry, the flame edge with the smaller density jump (the leaner flame edge) exhibits high amplitude, coherent wrinkling.

\section{ACKNOWLEDGMENTS}

This work has been partially supported by the National Science Foundation through Contract No. CBET-1235779 (contract monitor Professor Ruey-Hung Chen) and the Air Force Office of Scientific Research through Contract No. FA9550-12-1-0107/RC657 (contract monitor Dr. Chiping Lee).

\section{APPENDIX: STABILITY ANALYSIS FORMULATION DETAILS}

This appendix outlines the details of the stability analysis. The stability analysis assumes a normal modes decomposition for the linear flow perturbations in axial position, $x$, and in time, $t$. The linear perturbations to axial velocity, radial velocity, pressure, and stream function take the form

$$
u, v, p, \psi=\operatorname{Re}\{\hat{u}(y), \hat{v}(y), \hat{p}(y), \hat{\psi}(y)\} \exp \{-i \omega t+i k x\} .
$$

Here $\omega$ is the complex radial frequency, $k$ is the complex wave number, and the hat denotes a complex function of transverse position, $y$ (these "hat" quantities are referred to as the mode shapes). These mode shapes are modeled in the three base flow regions (see Figure 3) according to the Rayleigh equation, ${ }^{34}$ which is formulated here for top-hat base velocity profiles and linearly varying base density profiles

$$
\left(u_{x, 0}-c\right)\left(\frac{\partial^{2} \hat{\psi}_{1}}{\partial y^{2}}-k^{2} \hat{\psi}\right)=\frac{1}{\rho_{0}^{2}} \frac{\partial \rho_{0}}{\partial y}\left[\rho_{0} \hat{\psi}_{1} \frac{d u_{0}}{d y}-\rho_{0}\left(u_{0}-c\right) \frac{\partial \hat{\psi}_{1}}{\partial y}\right] .
$$

The general solution to this form of the Rayleigh equation gives the transverse dependence of the linear flow perturbations, which in regions of uniform base density is

$$
\hat{\psi}(y)=A e^{k y}+B e^{-k y}
$$

and in regions of linearly varying base density is

$$
\hat{\psi}(y)=A J_{0}\left(\frac{i k(b+a y)}{a}\right)+B Y_{0}\left(\frac{-i k(b+a y)}{a}\right) .
$$

In Eq. (A4), $J_{0}$ and $Y_{0}$ are zero-order Bessel functions of the first and second kind, and $a$ and $b$ define the linear density profile, $\rho_{b}(y)=a y+b$, depicted in Figure 3 . 
The dispersion relation for this analysis consists of six algebraic equations. These equations are derived from the boundary conditions at the channel walls and from matching conditions at the jump discontinuities in the base flow. The boundary condition at each wall is the impermeability condition. The matching conditions at the base flow jump discontinuities are continuity of displacement and continuity of hydrodynamic pressure.

Six equations are formulated to satisfy the impermeability condition at each channel wall, and to satisfy pressure and displacement matching conditions at the two base flow discontinuities. These equations, written in dimensional form, reference the base flow model shown in Figure 3. The subscripts attached to the coefficients $A$ and $B$ denote in which region of this model the coefficients govern the dynamics.

Impermeability at the lower channel wall (at $y=0)$

$$
A_{a}+B_{a}=0 .
$$

Impermeability at the upper channel wall (at $y=H$ )

$$
A_{c} \exp (k H)+B_{c} \exp (-k H)=0 .
$$

Pressure matching at the lower base flow discontinuity (at $y=\delta_{1}$ )

$$
\begin{gathered}
i k \rho_{b 1} U_{b} J_{1}\left(\frac{i k\left[b+a \delta_{1}\right]}{a}\right) A_{b}-i k \rho_{b 1} U_{b} Y_{1}\left(\frac{-i k\left[b+a \delta_{1}\right]}{a}\right) B_{b} \\
\rho_{a} U_{a} k \exp \left(k \delta_{1}\right) A_{a}-\rho_{a} U_{a} k \exp \left(-k \delta_{1}\right) B_{a}=\cdots \\
i k \rho_{b 1} J_{1}\left(\frac{i k\left[b+a \delta_{1}\right]}{a}\right) c A_{b}-i k \rho_{b 1} Y_{1}\left(\frac{-i k\left[b+a \delta_{1}\right]}{a}\right) c B_{b}+\cdots \\
k \rho_{a} \exp \left(k \delta_{1}\right) c A_{a}-k \rho_{a} \exp \left(-k \delta_{1}\right) c B_{a} .
\end{gathered}
$$

Displacement matching at the lower base flow discontinuity (at $y=\delta_{1}$ )

$$
\begin{gathered}
-U_{a} J_{0}\left(\frac{i k\left[b+a \delta_{1}\right]}{a}\right) A_{b}-U_{a} Y_{0}\left(\frac{-i k\left[b+a \delta_{1}\right]}{a}\right) B_{b}+U_{b} \exp \left(k \delta_{1}\right) A_{a}+U_{b} \exp \left(-k \delta_{1}\right) B_{a}=\cdots \\
-J_{0}\left(\frac{i k\left[b+a \delta_{1}\right]}{a}\right) c A_{b}-Y_{0}\left(\frac{-i k\left[b+a \delta_{1}\right]}{a}\right) c B_{b}+\exp \left(k \delta_{1}\right) c A_{a}+\exp \left(-k \delta_{1}\right) c B_{a} .
\end{gathered}
$$

Pressure matching at the upper base flow discontinuity (at $y=\delta_{2}$ )

$$
\begin{gathered}
\rho_{c} U_{c} k \exp \left(k \delta_{2}\right) A_{c}-\rho_{c} U_{c} k \exp \left(-k \delta_{2}\right) B_{c}+\cdots \\
i k \rho_{b 2} U_{b} J_{1}\left(\frac{i k\left[b+a \delta_{2}\right]}{a}\right) A_{b}-i k \rho_{b 2} U_{b} Y_{1}\left(\frac{-i k\left[b+a \delta_{2}\right]}{a}\right) B_{b}=\cdots \\
k \rho_{c} \exp \left(k \delta_{2}\right) c A_{c}-k \rho_{c} \exp \left(-k \delta_{2}\right) c B_{c}+\cdots \\
i k \rho_{b 2} J_{1}\left(\frac{i k\left[b+a \delta_{2}\right]}{a}\right) c A_{b}-i k \rho_{b 2} Y_{1}\left(\frac{-i k\left[b+a \delta_{2}\right]}{a}\right) c B_{b} .
\end{gathered}
$$

Displacement matching at the upper base flow discontinuity (at $y=\delta_{2}$ )

$$
\begin{gathered}
U_{b} \exp \left(k \delta_{2}\right) A_{c}+U_{b} \exp \left(-k \delta_{2}\right) B_{c}-U_{c} J_{0}\left(\frac{i k\left[b+a \delta_{2}\right]}{a}\right) A_{b}-U_{c} Y_{0}\left(\frac{-i k\left[b+a \delta_{2}\right]}{a}\right) B_{b}=\cdots \\
\exp \left(k \delta_{2}\right) c A_{c}+\exp \left(-k \delta_{2}\right) c B_{c}-J_{0}\left(\frac{i k\left[b+a \delta_{2}\right]}{a}\right) c A_{b}-Y_{0}\left(\frac{-i k\left[b+a \delta_{2}\right]}{a}\right) c B_{b} .
\end{gathered}
$$

The six equations, listed from Eqs. (A5)-(A10), are arranged as a generalized Eigenvalue problem with the phase speed, $c$, as the Eigenvalue and the Coefficients $A_{a}, B_{a}, A_{b}, B_{b}, A_{c}, B_{c}$, as the Eigenvector. This dispersion relation is solved by prescribing a value for the complex wave number, $k$, solving the Eigenvalue problem for $c$, and then calculating the complex frequency as $\omega=k / c$. The spatio-temporal stability problem proceeds by solving this dispersion relation and numerically 
searching for saddle points of $\omega(k)$. The Briggs-Bers validity 22,35 of the saddles is confirmed, and then the absolute frequency, growth rate, and wave number are defined at the most amplified, valid saddle, $\omega_{0}\left(k_{0}\right)$.

Setting the velocity and density asymmetry parameters to zero, i.e., $\chi_{u}=\chi_{\rho}=0$, and removing confinement, i.e., $H / D \rightarrow \infty$, recovers Yu and Monkewitz's result. ${ }^{25}$

${ }^{1}$ S. J. Shanbhogue, S. Husain, and T. Lieuwen, "Lean blowoff of bluff body stabilized flames: Scaling and dynamics," Prog. Energy Combust. Sci. 35, 98 (2009).

${ }^{2}$ D. A. Smith and E. E. Zukoski, "Combustion instability sustained by unsteady vortex combustion," AIAA Paper No. 85-1248, 1985.

${ }^{3}$ T. J. Poinsot, A. C. Trouve, D. P. Veynante, S. M. Candel, and E. J. Esposito, "Vortex-driven acoustically coupled combustion instabilities," J. Fluid Mech. 177, 265 (1987).

${ }^{4}$ M. C. Soteriou and A. F. Ghoniem, "The vorticity dynamics of an exothermic, spatially developing, forced reacting shear layer," Symp. (Intl.) Combustion 25(1), 1265 (1994).

5 J. E. Crump, K. C. Schadow, V. Yang, and F. E. C. Culick, "Longitudinal combustion instabilities in ramjet engines: Identification of acoustic modes," J. Propul. Power 2, 105 (1986).

${ }^{6}$ V. Yang and F. E. C. Culick, "Analysis of low frequency combustion instabilities in a laboratory ramjet combustor," Combust. Sci. Technol. 45, 1 (1986).

${ }^{7}$ W. O. Criminale, T. L. Jackson, and R. D. Joslin, Theory and Computation in Hydrodynamic Stability (The Press Syndicate of the University of Cambridge, Cambridge, 2003).

${ }^{8}$ G. S. Cardell, Flow Past a Circular Cylinder with a Permeable Splitter Plate (California Institute of Technology, 1993).

${ }^{9}$ A. Prasad and C. H. K. Williamson, "The instability of the shear layer separating from a bluff body," J. Fluid Mech. 333, 375 (1997).

${ }^{10}$ G. L. Brown and A. Roshko, "On density effects and large structure in turbulent mixing layers," J. Fluid Mech. 64, 775 (1974).

${ }^{11}$ C.-M. Ho and P. Huerre, Perturbed Free Shear Layers (University of Southern California, Los Angeles, 1984).

12 A. E. Perry, M. S. Chong, and T. T. Lim, “The vortex shedding process behind two-dimensional bluff bodies," J. Fluid Mech. 116, 77 (1982).

${ }^{13}$ B. Cantwell and D. Coles, "An experimental study of entrainment and transport in the turbulent near wake of a circular cylinder," J. Fluid Mech. 136, 321 (1983).

${ }^{14}$ R. D. Blevins, Flow-Induced Vibration (Van Nostrand Reinhold Co., New York, 1977).

15 A. Roshko, "On the wake and drag of bluff bodies," J. Aeronaut. Sci. 22, 124 (1955).

${ }^{16}$ R. F. Huang and K. T. Chang, "Oscillation frequency in wake of a vee gutter," J. Propul. Power 20, 871 (2004),

${ }^{17}$ B. Kiel, K. Garwick, A. Lynch, J. R. Gord, and T. Meyer, Non-Reacting and Combusting Flow Investigation of Bluff Bodies in Cross Flow (American Institute of Aeronautics and Astronautics, Sacramento, California, 2006), Vol. 5234.

${ }^{18}$ C. Cross, A. Fricker, D. Shcherbik, E. Lubarsky, B. T. Zinn, and J. A. Lovett, Dynamics of Non-Premixed Bluff BodyStabilized Flames in Heated Air Flow (American Society of Mechanical Engineers, Glasgow, UK, 2010), pp. 875-884.

${ }^{19}$ R. R. Erickson, M. C. Soteriou, and P. G. Mehta, The Influence of Temperature Ratio on the Dynamics of Bluff Body Stabilized Flames (American Institute of Aeronautics and Astronautics, Reno, Nevada, 2006), pp. 9-12.

${ }^{20}$ B. Emerson, J. O'Connor, M. Juniper, and T. Lieuwen, "Density ratio effects on reacting bluff-body flow field characteristics," J. Fluid Mech. 706, 219 (2012).

${ }^{21}$ R. R. Erickson and M. C. Soteriou, "The influence of reactant temperature on the dynamics of bluff body stabilized premixed flames," Combust. Flame 158, 2441 (2011).

${ }^{22}$ R. J. Briggs, Electron-Stream Interaction with Plasmas (MIT Press, Cambridge, MA, 1964).

${ }^{23}$ A. Bers, Handbook of Plasma Physics I: Basic Plasma Physics (North-Holland, Amsterdam, 1983).

${ }^{24}$ P. Huerre and P. A. Monkewitz, "Absolute and convective instabilities in free shear layers," J. Fluid Mech. 159, 151 (1985)

${ }^{25}$ M.-H. Yu and P. A. Monkewitz, "The effect of nonuniform density on the absolute instability of two-dimensional inertial jets and wakes," Phys. Fluids 2, 1175 (1990).

${ }^{26}$ B. Emerson, D. Noble, and T. Lieuwen, Stability Analysis of Reacting Wakes: The Physical Role of Flame-Shear Layer Offset (AIAA SciTech, National Harbor, Maryland, 2014).

${ }^{27}$ B. Kiel, K. Garwick, J. R. Gord, J. Miller, A. Lynch, R. Hill, and S. Phillips, A Detailed Investigation of Bluff Body Stabilized Flames (American Institute of Aeronautics and Astronautics, 2007), AIAA Paper No. AIAA, 168

${ }^{28}$ S. J. Shanbhogue, D.-H. Shin, S. Hemchandra, D. Plaks, and T. Lieuwen, Flame-Sheet Dynamics of Bluff-Body Stabilized Flames Using Longitudinal Acoustic Forcing (Elsevier, 2009).

${ }^{29}$ S. Tuttle, S. Chaudhuri, K. Kopp-Vaughan, T. Jensen, B. Cetegen, M. Renfro, and J. Cohen, "Lean blowoff behavior of asymmetrically-fueled bluff body-stabilized flames," Combust. Flame 160, 1677 (2013).

${ }^{30}$ P. Huerre and P. A. Monkewitz, "Local and global instabilities in spatially developing flows," Annu. Rev. Fluid Mech. 22, 473 (1990).

${ }^{31}$ K. R. Anderson, J. Hertzberg, and S. Mahalingam, "Classification of absolute and convective instabilities in premixed bluff body stabilized flames," Combust. Sci. Technol. 112, 257 (1996).

32 H. Schlichting, Boundary-Layer Theory (Springer, 2003).

${ }^{33}$ M. P. Juniper, O. L. Tammisola, and F. Lundell, "The local and global stability of confined planar wakes at intermediate Reynolds number," J. Fluid Mech. 686, 218 (2011).

${ }^{34}$ C. Godreche and P. Manneville, Hydrodynamics and Nonlinear Instabilities (Cambridge University Press, Cambridge, 1998).

35 A. Bers, Space-Time Evolution of Plasma Instabilities-Absolute and Convective (North-Holland, Amsterdam, 1983). 